\title{
Matérista
}

Revista Matéria, v. 15, n. 3, pp. 400 - 404, 2010

ISSN 1517-7076

http://www.materia.coppe.ufrj.br/sarra/artigos/artigo11123

\section{Compósitos biodegradáveis a base de polihidroxibutirato-hidroxivalerato (PHB-HV) reforçados com resíduos do beneficiamento do café}

\author{
Machado, A.R.T. ${ }^{\text {I; }}$ Martins, P.F.Q. ${ }^{\text {I; }}$ Fonseca, E.M.B. ${ }^{\text {I' }}$ Reis, K.C. ${ }^{\text {II }}$ \\ I Departamento de Química-DQI, Universidade Federal de Lavras-UFLA, CEP: 37200-000 - Lavras, MG. \\ e-mail: alanvisconde@gmail.com ; martinspfq@gmail.com ; emanuella.fonseca@gmail.com \\ ${ }^{\text {II }}$ Departamento de Engenharia de Materiais - DEMa , Universidade Federal de São Carlos -UFSCar, CEP: \\ 13565-905, São Carlos, SP. \\ e-mail: kelen_cr@yahoo.com.br
}

\section{RESUMO}

Compósitos de polihidroxibutirato - hidroxivalerato (PHB-HV) reforçados com resíduos da produção de café foram preparados a partir de PHB-HV com dois diferentes tipos de resíduos, o pergaminho e a casca de café, através da técnica de evaporação de solvente "casting". Para avaliar o efeito dos resíduos na matriz, os compósitos foram avaliados por difração de raios x (DRX), Microscopia eletrônica de Varredura (MEV) e através das propriedades mecânicas. Estas técnicas forneceram fortes evidencias da formação de uma estrutura intercalada e homogênea entre os precursores, sem que isto alterasse a cristalinidade do PHBHV. As propriedades mecânicas foram avaliadas quanto à força de punctura e deformação antes da ruptura indicando que a presença da carga orgânica eleva ambas as propriedades, gerando um novo material com um campo de aplicação ampliado em relação ao PHB-HV.

Palavras-chaves: PHB-HV, compósitos, resíduos agroindustriais, casca e pergaminho de café.

\section{Biodegradable polyhydroxybutyrate-hydroxyvalerate (PHB-HV) composites reinforced with residue of processing coffee}

\section{ABSTRACT}

Polyhydroxybutyrate-hydroxyvalerate (PHB-HV) coffee residue composites were prepared from PHB-HV and two different loadings of residue the husk and pill of coffee. The solvent evaporation technique" casting" was used to form the films. To evaluate the residue effect in the PHB-HV properties and the material dispersion, the films were analyzed by X-ray diffraction (XRD), Scanning Electronic Microscopy (SEM) and from mechanical properties. These techniques provided strong evidence that the residue formed a homogeneous intercalated structure, although it did not altered the cristalinity of PHB-HV. The mechanical properties were evaluated by puncture force and deformation before the rupture, indicating that the presence of the organic charge elevates both properties. The composite obtained is a new material with a wider application field than the PHB-HV.

Keywords: PHB-HV, composites, agro industrial waste, coffee husk and peel.

\section{INTRODUÇÃO}

A utilização de fibras sintéticas para o reforço de polímeros é uma técnica extensivamente empregada na indústria para a obtenção de materiais com melhor desempenho mecânico, no entanto a busca por materiais não agressivos ao meio ambiente, o desenvolvimento de compósitos poliméricos envolvendo o emprego de fibras vegetais e polímeros naturais vêm crescendo cada vez mais [1].

As fibras naturais são adicionadas a matriz polimérica visando melhorar suas propriedades e reduzir custos da composição polimérica e/ou a geração de efluentes [2]. A utilização das fibras vegetais, tais como juta, algodão, sisal e coco, como reforço em comparação às cargas inorgânicas possui muitas vantagens, tais como: obtenção de materiais de baixa densidade, menor abrasão durante processamento, altos níveis de preenchimento que resultam em aumento na rigidez, elevado módulo específico, aumento na durabilidade [3]. Além disso, as fibras naturais são biodegradáveis, provenientes de fontes renováveis de grande disponibilidade e de baixo custo. 
Os poli (hidroxialcanoatos) (PHAs) são poliésteres produzidos por processos biossintéticos, sendo que o poli(3-hidroxibutirato) (PHB) e seu copolímero polihidroxibutirato-hidroxivalerato (PHB-HV) figura 1 tem despertado interesse comercial, devido à possibilidade de produção por rotas biotecnológicas a partir de substrato renovável e por apresentar-se como uma alternativa à substituição dos plásticos convencionais derivados do petróleo, os quais possuem lenta degradação, provocando acúmulo de resíduos sólidos contaminando o ambiente [4].

O PHB é produzido como material de reserva energética por fermentação de uma variedade de cepas bacterianas, destacando-se a Ralstonia eutropha e a Burkholderia saccharina. É um poliéster semicristalino, com grande potencial como polímero biodegradável de alto consumo. A sua degradação ocorre por erosão da superfície [ $[\underline{]}$.

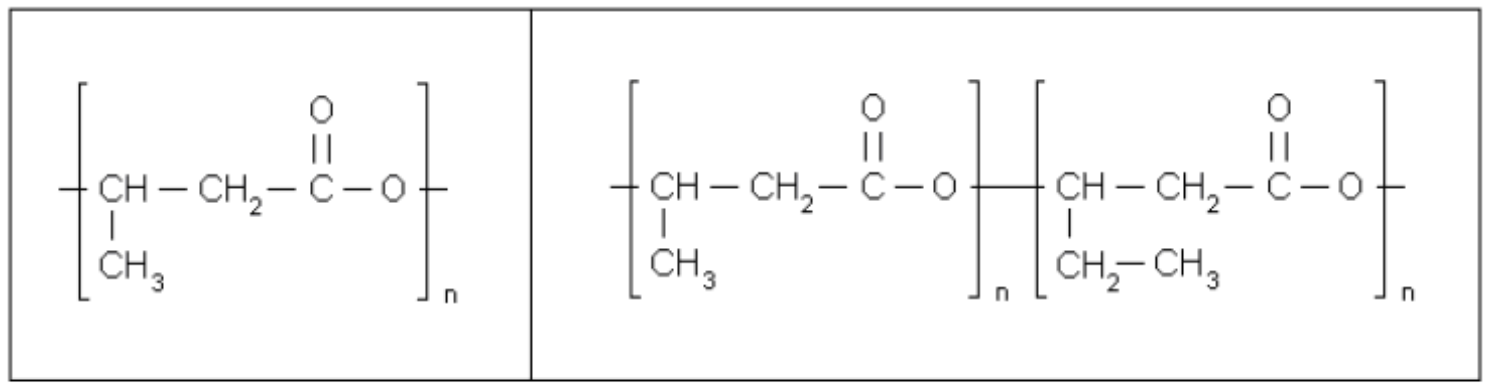

(a)

(b)

Figura 1: Estrutura química do PHB (a) e do seu copolímero PHB-HV (b).

A cafeicultura dá origem a um volume elevado de resíduos, principalmente a casca de café, cuja utilização tem sido objeto de estudos [6]. A polpa, mucilagem, pergaminho e casca são resíduos oriundos de formas distintas do beneficiamento do café após a colheita. No Brasil, a forma mais comum de preparo do café é por via seca, em que o fruto é seco na sua forma integral, resultando em resíduos formados por casca e pergaminho, fornecendo um rendimento de $50 \%$ do peso colhido. A grande maioria das pesquisas realizadas com resíduos do café foi feita com a polpa e, em menor número, com a casca ou pergaminho, no entanto as composições da casca e da polpa são semelhantes [7].

Diante do exposto o objetivo deste trabalho foi elaborar compósitos biodegradáveis reforçados com os resíduos do beneficiamento do café, casca e pergaminho, e caracterizar os compósitos através de difração de raios $\mathrm{x}$, microscopia eletrônica de varredura e análise mecânica.

\section{MATERIAIS E MÉTODOS}

\subsection{Materiais}

O polihidroxibutirato-Hidroxivalerato (PHB-HV) foi fornecido pela PHB Industrial S/A, Serrana, São Paulo. O Clorofórmio utilizado foi de grau analítico (marca Vetec). A casca e o pergaminho foram obtidos da colheita do final de junho e inicio de agosto de 2007, das fazendas da Epamig (Machado-MG).

\subsection{Método}

Foram preparados compósitos Casca e Pergaminho em matrizes de PHB-HV na proporção de 1:1 utilizando o método de evaporação de solvente 'casting', técnica amplamente citada na literatura $[\underline{8}, \underline{9}, \underline{10}]$.

Para preparação dos compósitos submeteu-se o PHB-HV e Casca ou Pergaminho a aquecimento com clorofórmio em frasco fechado sob agitação magnética por 45 minutos, obtendo-se uma mistura polimérica a $5 \%(\mathrm{~m} / \mathrm{m})$. A mistura foi então vertida numa placa de petri, que em seguida foi colocada em uma cuba de vidro totalmente fechada para a lenta evaporação do solvente, por 24 horas. O compósito obtido foi seco em estufa a vácuo à $60^{\circ} \mathrm{C}$ por 12 horas e depois armazenado em sacos plásticos de polietileno.

Os compósitos obtidos foram caracterizados por microscopia eletrônica de varredura (MEV) em um microscópio de varredura LEO EVO 40XVP, difração de raios x, equipamento da marca Philips, com variação angular de $10-70^{\circ}(2 \theta)$, radiação de $\operatorname{CoK} \alpha(\lambda=1.78897 \AA)$ e velocidade de exposição de $1^{\circ} 20 \min ^{-1}$. A resistência mecânica foi avaliada através de força de punctura através do analisador de textura TATX.2i Stable Micro Systems (Goldaming, England). Amostras com $60 \mathrm{~mm}$ de diâmetro foram fixadas com fita adesiva em uma célula de perfuração com diâmetro de $20 \mathrm{~mm}$. Uma probe cilíndrica de $6 \mathrm{~mm}$ de diâmetro foi 
movida perpendicularmente a uma distância de $30 \mathrm{~mm}$ em direção à superfície do compósito a uma velocidade de pré-teste de $1 \mathrm{~mm} / \mathrm{s}$ e velocidade de teste de $1 \mathrm{~mm} / \mathrm{s}$ até o rompimento da superfície do compósito. O valor da força de punctura $(\mathrm{N})$ foi determinado a partir de curvas de força $(\mathrm{N}) \mathrm{x}$ deslocamento $(\mathrm{mm})$ da probe obtidas com o uso do Software Texture Expert Versão 1.22, 1999. Empregaram-se dez repetições e os valores foram submetidos à análise de variância (ANAVA), empregando-se o programa computacional SISVAR. As médias foram comparadas segundo o teste de Scott \& Knott (1974) [11] a $5 \%$ de probabilidade

\section{RESULTADOS E DISCUSSÃO}

A Microscopia eletrônica evidencia a estrutura porosa do PHB-HV (Figura 2a), no entanto essa característica é fortemente reduzida nos compósitos (Figuras $2 \mathrm{~b}$ e $2 \mathrm{c}$ ) o que visivelmente esta relacionada com a presença dos resíduos nos espaços antes vazios da estrutura. Os espaços vazios remanescentes podem ser poros não preenchidos, zonas em que se acumularam gases ou até mesmo locais onde a partículas não se aderiram corretamente à matriz de PHB-HV.

A análise das micrografias permite a conclusão de que os compósitos de $\mathrm{PHB}-\mathrm{HV} /$ pergaminho e PHB-HV/ casca apresentaram boa dispersão dos resíduos de café por todo o filme e estes estão bem aderidos à matriz de PHB-HV.

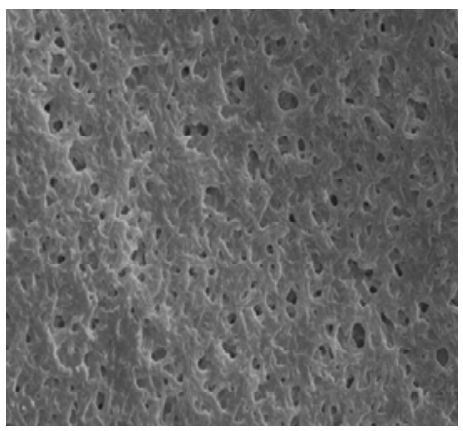

(a) $20 \mu \mathrm{m}$

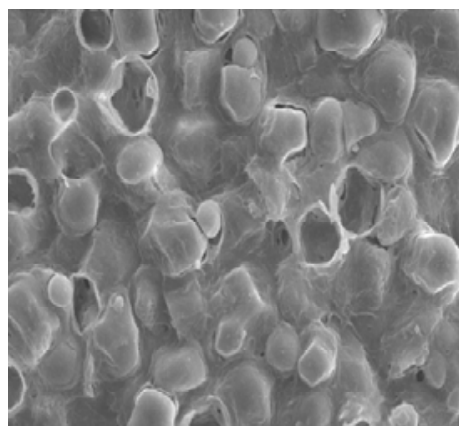

(b) $10 \mu \mathrm{m}$

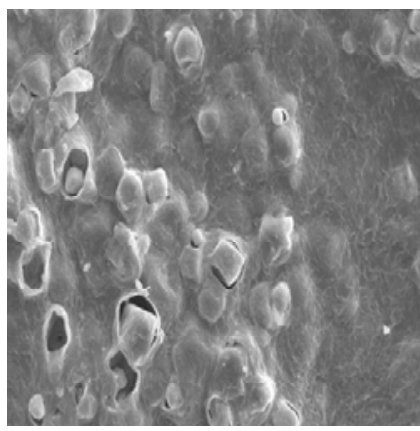

(c) $10 \mu \mathrm{m}$

Figura 2: Micrografias da morfologia do PHB-HV (a) e dos compósitos PHB-HV/ pergaminho (b) e PHB$\mathrm{HV} / \operatorname{casca}(\mathrm{c})$.

Os difratogramas de raios-X dos compósitos (Figura 3) indicam que os diferentes materiais utilizados na elaboração dos compósitos não influenciaram a estrutura cristalina do PHB-HV puro, considerando que os valores das distâncias interplanares e a largura das reflexões cristalinas foram similares aos relatados na literatura para o PHB-HV puro [12], apresentando reflexões principais em $2 \theta=17,19$ e $30^{\circ}$. Este resultado sugere que a biodegradabilidade do material não foi afetada pela adição dos resíduos [13]

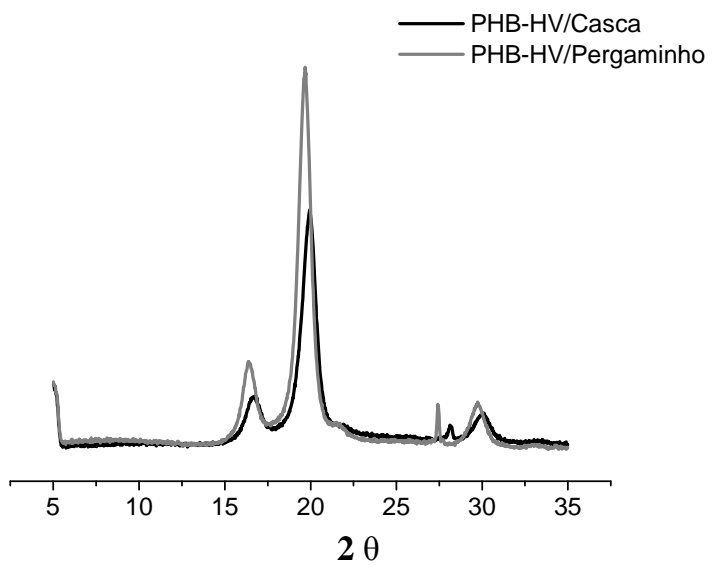

Figura 3: Difratogramas de raio-X dos compósitos de PHB-HV e resíduos do beneficiamento de café. 
As propriedades mecânicas dos compósitos dependem de diversos fatores, tais como o tipo, volume, orientação e dispersão da fibra na matriz, bem como a afinidade química entre matriz e fibra [14]. As propriedades mecânicas apresentaram um aumento significativo com a incorporação dos resíduos (Tabela 1) sugerindo uma boa dispersão destes na matriz de PHB-HV, como se pode observar nas imagens de microscopia eletrônica de varredura (Figura 2). Observa-se na Tabela 1 que não ocorreu diferença estatística entre os compósitos obtidos com pergaminho e casca de café sendo que ambos resultaram em aumento da força de punctura e da deformação na punctura. A presença dos resíduos dispersos homogeneamente na matriz funcionou como um reforço estrutural para o PHB-HV cuja estrutura é bastante porosa (Figura 2) e quebradiça [14]. Wambua [15] estudando algumas fibras vegetais, como coco, sisal, juta, abacaxi e linho, para substituição das fibras de vidro como reforço de terrmoplasto, também obteve aumento nas propriedade mecânicas dos compósitos, tendo o coco apresentado o menor valor de propriedade mecânica nos ensaios realizados e o linho o maior desempenho.

Tabela 1: Força de punctura e deformação na punctura do PHB-HV e seus compósitos com pergaminho e casca de café.

\begin{tabular}{ccc}
\hline Materiais & Força de punctura (N) & Deformação na punctura (\%) \\
\hline PHB-HV & $8,12 \mathrm{a}$ & $3,30 \mathrm{c}$ \\
PHB-HV/ pergaminho de café & $9,98 \mathrm{~b}$ & $5,60 \mathrm{~d}$ \\
PHB-HV/ casca de café & $9,63 \mathrm{~b}$ & $5,29 \mathrm{~d}$ \\
\hline
\end{tabular}

* Médias seguidas pela mesma letra não diferem significativamente entre si pelo teste de Scott \& Knott a 5\% de significância.

\section{CONCLUSÕES}

Neste trabalho investigou-se o efeito da incorporação da casca e do pergaminho de café na matriz de PHB-HV. Os compósitos obtidos apresentam-se como uma boa alternativa ao PHB-HV puro, pois a presença dos resíduos elevou as propriedades mecânicas do material sem interferir significativamente em sua cristanilidade, gerando uma destinação ecologicamente correta, e de alto valor agregando, para os resíduos do beneficiamento do café. A inserção dos resíduos permitiu ainda a diminuição da massa de polímero utilizada para confecção de produtos o que amplia consideravelmente o número de aplicações para o polímero biodegradável em questão. Os resultados mostram que resíduos de beneficiamento de café podem ser empregados como reforços em compósitos de matrizes poliméricas biodegradáveis.

\section{AGRADECIMENTOS}

Os autores agradecem a FAPEMIG e a Universidade Federal de Lavras - UFLA.

\section{BIBLIOGRAFIA}

[1] HRISTOV, V.N., LACH, R., GRELlMANN, W., "Impact fracture Behavior of modified polypropylene/wood fiber composites", Polymer Testing, v. 23, pp. 381-389, 2004.

[2] MARTINES, M.A., "Fibras de Sisal: Mercerização, Acetilação e Aplicação em Compósitos de Borrocha de Pneu Triturado", Tese de D.Sc., Instituto de Química, Universidade Estadual de Campinas, Campinas, 2001.

[3] TITA, S.P.S., PAIVA, J.M.F., FROLLINI, E., "Resistência ao impacto e outras propriedades de compósitos lignocelulósicos: matrizes termofixas fenólicas reforçadas com fibras de bagaço de cana-de-açúcar", Polímero: Ciência e Tecnologia, v. 12, pp.228-239, 2002.

[4] SANTOS, A.F., "Caracterização, modificação e estudo cinético não isotérmico de poli(3hidroxibutirato)", Dissertação de M.Sc, UNESP ,São Paulo, SP, 2005.

[5] FARAGO, P.V., SÁ, B.C.M., PERUSSE, C.A., et al., "Micropartículas de poli (hidroxibutirato-cohidroxivalerato) como sistema de liberação de controlada de metformina: obtenção e caracterização", In: 17 Congresso Brasileiro de Ciências e Engenharia de Materiais, Foz do Iguaçu, 2006. 
[6] VEGRO, C.L.R., CARVALHO, F.C., "Disponibilidade e utilização de resíduos no processamento agroindustrial do café”, Informações Econômicas, v. 24, n. 1, pp.9-16, jan. 1994.

[7] CAIELLI, E.L., "Uso da palha de café na alimentação de ruminantes”, Informe Agropecuário, Belo Horizonte, v. 10, n. 119, pp. 36-38, nov.1984.

[8] VANIN, M., SANTANA, C.C., DUEK, E.A.R., TORRIANI, I.L., PRIVELIC, T., "Estudo da degradação "in vitro" de blendas de poli(beta-hidroxibutirato) (PHB) / poli(L-ácido láctico) (PLLA) na forma de filmes", Polímeros - Ciência e Tecnologia, v. 14, n. 3, pp. 187-193, 2004.

[9] SCHMIDT, V., DOMENECH, S.C., SOLDI, M.S., PINHEIRO,E.A., SOLDI, V., "Thermal stability of polyaniline/ethylene propylene diene rubber blends prepared by solvent casting", Polymer Degradation and Stability, v. 83, pp. 519 , 2004.

[10] YONG, K.C., FOOT, P.J.S., MORGAN, H., "Conductive poly(butadiene-co-acrylonitrile)-polyaniline dodecylbenzenesulfonate [NBR-PAni.DBSA] blends prepared in solution”, European Polylimer Journal, v. 42, pp. 1716, 2006.

[11] SCOTT, A.J., KNOTT, M.A., "A cluster analysis method for grouping means in the analysis of variance", Biometrics, v. 30, n. 2, pp. 507-512, 1974.

[12] REIS, K.C., PEREIRA, J., SMITH, A., CARVALHO, C.W.P., WERNER, N.; IRYNA, A. "Characterization of polyhydroxybutyrate-hydroxyvalerate (PHB-HV)/ maize starch blend films", Journal of Food Engineering, v. 89, pp. 361-369, 2008.

[13] SPINACÉ, M.A.S., LAMBERT, C.S., FERMOSELLI, K.K.G., PAOLI, M.A., "Characterization of lignocellulosic curaua fibres”, Carbohydrate Polymers, v. 77, n. 1, pp. 47-53, 2009.

[14] COELHO, N.S., ALMEIDA, Y.M.B., VINHAS, G.M., "A biodegradabilidade da blenda de poli( $\beta$ Hidroxibutirato-co-Valerato)/amido anfótero na presença de microorganismos”, Polímeros, v. 18, n. 3,2008

[15] WAMBUA, P., IVENS, J., VERPOEST, I., "Natural Fibers: can they replace glass in fibre reiforced plastics?”, Composites Science and Technology, v. 63, n. 9, pp. 1259-1264, 2003. 